\title{
GATA4 protects granulosa cell tumors from TRAIL-induced apoptosis
}

\author{
Antti Kyrönlahti, 1,2,6*, Marjut Kauppinen ${ }^{1,2 *}$, Essi Lind ${ }^{5}$, Leila Unkila-Kallio ${ }^{2,3}$, \\ Ralf Butzow ${ }^{2,4}$, Juha Klefström ${ }^{5}$, David B Wilson ${ }^{6}$, Mikko Anttonen $^{2,3}$ \\ and Markku Heikinheimo ${ }^{1,2,6}$
}

\footnotetext{
${ }^{1}$ Children's Hospital, University of Helsinki, PO Box 22 (Stenbäckinkatu 11), 00014 Helsinki, Finland

${ }^{2}$ Program for Women's Health, ${ }^{3}$ Department of Obstetrics and Gynecology, Biomedicum Helsinki, ${ }^{4}$ Department of Pathology and

${ }^{5}$ Institute of Biomedicine and Research Program in Genome-Scale Biology, University of Helsinki, 00014 Helsinki, Finland

${ }^{6}$ Department of Pediatrics, Washington University School of Medicine, St Louis, Missouri 63110, USA
}

(Correspondence should be addressed to M Heikinheimo at Children's Hospital, University of Helsinki;

Email: markku.heikinheimo@ helsinki.fi)

*(A Kyrönlahti and M Kauppinen contributed equally to this work)

\begin{abstract}
Disturbances in granulosa cell apoptosis have been implicated in the pathogenesis of human granulosa cell tumors (GCTs). Tumor necrosis factor-related apoptosis-inducing ligand (TRAIL) is a potent cytokine that induces apoptosis in a variety of malignancies without toxic effects on benign cells. The aim of this study was to investigate the expression and functionality of the TRAIL receptors DR4 and DR5 in human GCTs. Additionally, we examined the role of GATA4, a transcription factor expressed in normal and malignant granulosa cells, in TRAIL-induced GCT apoptosis. For this purpose, a tissue microarray of 80 primary and 12 recurrent GCTs was subjected to immunohistochemistry for DR4 and DR5, and freshly isolated primary GCT cultures were utilized to evaluate the functional effects of TRAIL on GCT cells. To clarify the role of GATA4 in the regulation of TRAIL-induced apoptosis, a human GCT-derived cell line (KGN) was transduced with lentiviral vectors expressing small hairpin RNAs targeting GATA4 or transfected with adenovirus expressing either wild-type or dominant negative mutant GATA4. We found that receptors DR4 and DR5 are expressed in a vast majority of GCTs as well as in primary GCT cultures, and that TRAIL induces apoptosis in the primary GCT cultures. Moreover, we showed that overexpressing GATA4 protects GCTs from TRAIL-induced apoptosis in vitro, whereas disrupting GATA4 function induces apoptosis and potentiates the apoptotic effect of TRAIL administration. Our results demonstrate that the TRAIL pathway is functional in GCT cells, and suggest that transcription factor GATA4 may function as a survival factor in this ovarian malignancy.
\end{abstract}

Endocrine-Related Cancer (2010) 17 709-717

\section{Introduction}

Human ovarian granulosa cell tumors (GCTs), accounting for $5 \%$ of all ovarian cancer, are the most common sex chord-derived malignancies with an incidence from 0.4 to 1.7 per 100000 women (Schumer \& Cannistra 2003, Jamieson \& Fuller 2008). GCTs typically present with relatively low malignant potential. However, these tumors can relapse decades after primary diagnosis, and the treatment of recurrent disease is challenging (Schumer \& Cannistra 2003). Platinum-based chemotherapy has been used in these tumors, but novel-targeted treatments are needed especially in the recurrent cases (Colombo et al. 2007). Thus, unraveling the exact mechanisms leading to GCT development is needed in the search for new therapies. Recently, a mutation in the gene encoding for the transcription factor FOXL2 has been found in a vast majority of GCTs studied (Shah et al. 2009), but the impact of this alteration remains unknown.

Disturbances in the regulation of granulosa cell apoptosis are suggested to play a role in granulosa cell tumorigenesis (Fuller \& Chu 2004). This is plausible given that the physiological fate of most normal 
granulosa cells is apoptosis. Successful follicular development gives rise to typically only one oocytecontaining follicle per cycle, while majority of the initially recruited follicles degenerate through apoptosis (Tilly 1996). In general, apoptosis is regulated by two interlinked mechanisms: extrinsic and intrinsic pathways (Fulda \& Debatin 2006). In ovaries, the physiological importance of Bcl-2 family in regulating the intrinsic pathway has been extensively studied (Pru \& Tilly 2001). Granulosa cell apoptosis is compromised in transgenic mice overexpressing the anti-apoptotic factor Bcl-2 (Hsu et al. 1996), whereas the number of follicles is reduced in Bcl-2 null mice (Ratts et al. 1995). Recently, we have demonstrated high level expression of Bcl-2 in a majority of human GCTs, suggesting a role for Bcl-2 in the regulation of apoptosis in these tumors (Kyronlahti et al. 2008).

The ligands of the extrinsic pathway, especially tumor necrosis factor-related apoptosis-inducing ligand (TRAIL), have emerged as potent cytokines that induce programmed cell death in a variety of human malignancies without significant toxicity for normal cells (Johnstone et al. 2008). The effects of TRAIL are mediated by two functional cell surface receptors, DR4 (also known as TRAIL-R1 and TNFRSF10A) and DR5 (TRAIL-R2 and TNFRSF10B). In humans, DR5 is expressed both in fetal and in adult granulosa cells (Jaaskelainen et al. 2009). The same study reported no DR4 expression in adult or fetal granulosa cells; however, we have detected modest DR4 staining also in adult granulosa cells (data not shown). The expression of TRAIL receptors in human GCTs is not well documented, although recombinant TRAIL is known to induce apoptosis in human granulosa cell line KGN (Woods et al. 2008a,b, Jaaskelainen et al. 2009).

We herein demonstrate that the TRAIL receptors DR4 and DR5 are expressed in a majority of human GCTs, and that TRAIL can effectively induce apoptosis in isolated primary tumor cells. Furthermore, we show that GATA4 (named after its binding to a consensus W-GATA-R motif), a transcription factor implicated in ovarian apoptosis and GCT pathobiology (Heikinheimo et al. 1997, Anttonen et al. 2005, Kyronlahti et al. 2008), can protect tumor cells from TRAIL-induced apoptosis in vitro, whereas inhibiting GATA4 function sensitizes tumor cells to TRAILinduced apoptosis. Finally, we show that disturbing GATA4 function alone is sufficient to induce apoptosis in vitro, suggesting that GATA4 may function as a survival factor in GCTs. Inhibition of GATA4 combined with TRAIL administration emerges as a potential means for targeted treatment of GCTs.

\section{Materials and methods}

\section{Human tissue samples}

This study was approved by the ethical committee of Helsinki University Central Hospital and the National Supervisory Authority for Welfare and Health in Finland. GCT tissue microarray of quadruple samples from 80 primary and 12 recurrent GCT samples was constructed as previously described (Kononen et al. 1998, Anttonen et al. 2005). For primary cell cultures (see below), informed patient consent was obtained before surgical operations. We obtained six fresh tumor samples, two from primary tumors and four from recurrent tumors, and all of them were verified to possess the $402 \mathrm{C} \rightarrow \mathrm{G}(\mathrm{C} 134 \mathrm{~W})$ mutation in FOXL2 (Shah et al. 2009). All the GCT diagnoses were re-evaluated, and tumor subtype, mitotic index, and nuclear atypia were defined by an expert pathologist (R B) as previously described (Anttonen et al. 2005); full clinical data of the patients were reviewed from hospital files.

\section{Immunohistochemistry}

Immunohistochemical staining of GCT tissue microarrays was performed as previously described (Anttonen et al. 2005, Kyronlahti et al. 2008). The primary antibodies used were as follows: goat antihuman DR4 IgG at 1:100 dilution (sc-6823, Santa Cruz Biotechnology, Inc., Santa Cruz, CA, USA); goat antihuman DR5 IgG at 1:50 dilution (sc-7192, Santa Cruz Biotechnology). Tissue samples stained with secondary antibody alone served as a negative control.

The immunohistochemistry results were classified into three groups based on the intensity of staining as follows: low ( - ), intermediate $(+)$, and strong $(++)$. Two researchers (A K and $\mathrm{M} \mathrm{K})$ independently performed the evaluation, and disagreements were resolved by a joint review with a third researcher (M A).

\section{Cell cultures and stimulations}

Following intraoperative diagnosis of GCT, tumor tissue was immediately obtained for cell culture. GCT tissue was mechanically minced and treated with $0.5 \%$ collagenase (Sigma-Aldrich Corporation) in DMEM/F12 containing 0\% fetal bovine serum (FBS) for $2 \mathrm{~h}$. The resultant cell suspension was then filtered through $140 \mu \mathrm{m}$ filter mesh to obtain single cells, washed with PBS, and re-suspended in DMEM/F12 containing $10 \%$ FBS. Finally, the primary tumor cells were counted and plated for experiments. 
The KGN cell line (a generous gift from Dr Toshihiko Yanase, Kyushu University, Fukuoka, Japan), established from a human GCT, was cultured as previously described (Nishi et al. 2001). This cell line harbors the $402 \mathrm{C} \rightarrow \mathrm{G}$ mutation in FOXL2 found in a majority of human GCTs (Schrader et al. 2009). To induce apoptosis, the cells were incubated with recombinant human TRAIL (Millipore, Bedford, MA, USA) at concentrations of 10,50 , and $100 \mathrm{ng} / \mathrm{ml}$. After 6-24 $\mathrm{h}$ incubation, the apoptosis was analyzed by activated caspase- 3 western blotting (see below), 4',6-diamino-2-phenylindole hydrochloride (DAPI) staining (see below), and Caspase-Glo 3/7 assay (see below).

\section{Immunocytochemistry and western blotting}

Primary GCT and KGN cells were plated on 8-well glass chambers, and then confluently washed with PBS and fixed with $4 \%$ paraformaldehyde for $20 \mathrm{~min}$ at room temperature. Immunocytochemical staining was performed using Vectastain Elite ABC kits (Vector Laboratories, Burlingame, CA, USA). Primary antibodies were as follows: mouse antihuman a-inhibin IgG (MCA 9515, AbD Serotec, Oxford, UK) at dilution of 1:100, goat antihuman antiMüllerian hormone (AMH) IgG (sc-6886, Santa Cruz Biotechnology, Inc.) at dilution of 1:100, and goat antihuman GATA4 IgG (sc-1237, Santa Cruz Biotechnology) at dilution of 1:200; goat antihuman DR4 IgG at 1:100 dilution (sc-7863, Santa Cruz Biotechnology), goat antihuman DR5 IgG at 1:50 dilution (sc-7192, Santa Cruz Biotechnology), rabbit antihuman DR4 IgG at 1:400 dilution (ab8414, Abcam, Cambridge, UK), and DR5 IgG at 1:400 dilution (ab8416, Abcam).

Western blotting was performed as previously described (Kyronlahti et al. 2008). Antibodies used were as follows: rabbit antihuman cleaved caspase-3 IgG (\#9661L, Cell Signaling Technology, Danvers, MA, USA) at dilution of 1:1000, goat antihuman GATA4 IgG (sc-1237, Santa Cruz Biotechnology) at dilution of 1:1000, and goat antihuman $\beta$-actin IgG (sc-1616, Santa Cruz Biotechnology, Inc.) at dilution of $1: 10000$.

\section{Caspase activation assay and DAPI analysis}

Caspase-3/7 activation was measured using CaspaseGlo 3/7 assays (Promega Corporation) as previously described (Jaaskelainen et al. 2009). For DAPI staining, cells were washed twice with PBS and fixed with formalin for $10 \mathrm{~min}$ at room temperature. After rinsed with PBS, cells were incubated with cold methanol for $10 \mathrm{~min}$ at $-20^{\circ} \mathrm{C}$, washed with PBS, and incubated for $30 \mathrm{~min}$ at room temperature with $0.4 \mu \mathrm{g} / \mathrm{ml}$ of DAPI in PBS. Finally, the cells were dehydrated through graded alcohols and Ultra Clear (J T Baker, Deventer, Holland), and mounted with UltraKitt (J T Baker).

\section{Adenoviral transfection}

Adenoviral transfections were performed as previously described (Kyronlahti et al. 2008). The replicationdeficient adenoviral construct expressing wild-type rat GATA4 (G4wt) and dominant negative GATA4 (G4dn, GATA4 producing engrailed repressor domain fusion protein tagged with flag epitope) have been previously described (Charron et al. 2001, Liang et al. 2001).

\section{Generation of GATA4 small hairpin RNA expressing stable cell lines}

pLKO.1 small hairpin RNA (shRNA) targeting GATA4 (shGATA4)-containing constructs were obtained from Sigma (TRC shRNA lentivirus library) and were as follows: shGATA4-1 (TRCN0000020426) 5'-GAGGAGATGCGTCCCATCAA-3' and shGATA4-2 (TRCN0000020427) 5'-CTGAATAAATCTAAGACACCA- $3^{\prime}$. Lentiviruses were produced as previously described (Partanen et al. 2007) using packaging constructs and protocols provided by the Biomedicum Helsinki Virus Core Facility.

Subconfluent 6-well plates of KGN cells were transduced with $750 \mu \mathrm{l}$ of lentiviral particles in a total volume of $1.5 \mathrm{ml}$ in DMEM/F12 containing $10 \%$ FBS. Polybrene was added to enhance transduction at 1:1000 dilution. Cells were incubated with lentiviral supernatant for $10 \mathrm{~min}$ at $+37^{\circ} \mathrm{C}$, centrifuged at 2500 r.p.m $(500 \mathrm{~g})$. for $30 \mathrm{~min}$, and then incubated at $+37^{\circ} \mathrm{C}$ for $2 \mathrm{~h}$, after which the lentiviral supernatant was replaced with normal growth media. After $72 \mathrm{~h}$, 1:2500 transduced cells were selected with puromycin $(1 \mu \mathrm{g} / \mathrm{ml})$.

\section{Statistical analysis of the data}

For tissue microarray scoring results and clinicopathological data, contingency tabling $(2 \times 2)$ was performed for the nominal variables, followed by the $\chi^{2}$ or Fisher's exact test. Statistical analysis of caspase assay experiments was performed using the Student's $t$-test (for G4dn and G4wt experiments) or one-way ANOVA, followed by Dunnett's test (for shGATA4 experiments) using JMP software (JMP, Cary, NC, USA). $P<0.05$ was considered significant in all experiments. 


\section{Results}

\section{TRAIL receptors are expressed in human GCTs}

To study the expression of the TRAIL receptors in GCTs, a tissue microarray based on 80 primary and 12 recurrent GCT samples was subjected to immunohistochemistry for DR4 and DR5. Of the GCTs analyzed, $91 \%$ exhibited strong (18\%) or intermediate (73\%) immunostaining for DR4 (using sc-6823 antibody); only 9\% exhibited low DR4 expression (Fig. 1A and C). Similarly, strong or intermediate DR5 expression was detected in $92 \%$ of the GCTs (strong $17 \%$ and intermediate $75 \%$ ), and only $8 \%$ of the tumors exhibited low DR5 expression (Fig. 1B and D).

To validate DR4 staining results, we utilized a second commercially available antibody (ab8414). These results significantly correlated $(P<0.001)$ with those of the sc-6823 DR4 antibody. However, we were unable to validate DR5 stainings (sc-7192) with another antibody (ab8416); these stainings remained unreliable given that we found nuclear staining in addition to membrane staining when using this antibody.
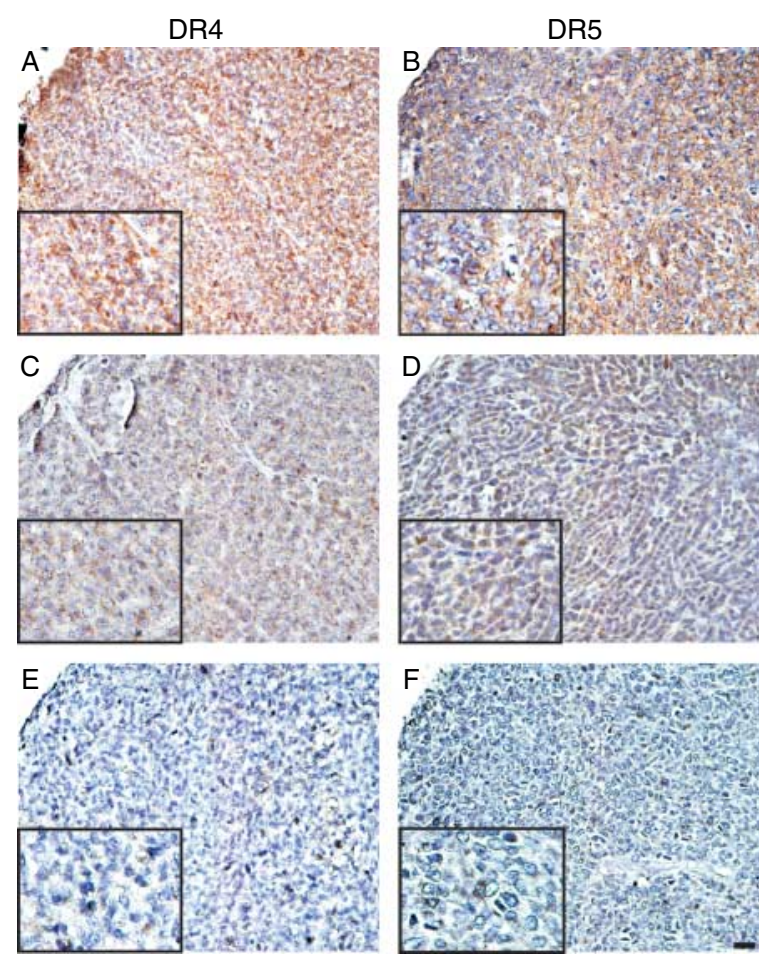

Figure 1 Immunohistochemical analysis of death receptors $\mathrm{DR} 4(\mathrm{~A}, \mathrm{C}$, and $\mathrm{E})$ and DR5 (B, D, and F) from tissue microarray of GCTs. Examples of strong, intermediate, and low expression are depicted in upper, middle, and lower rows respectively. Higher magnifications are shown in insets. Brown color indicates positive staining. Scale bar is $50 \mu \mathrm{m}$.
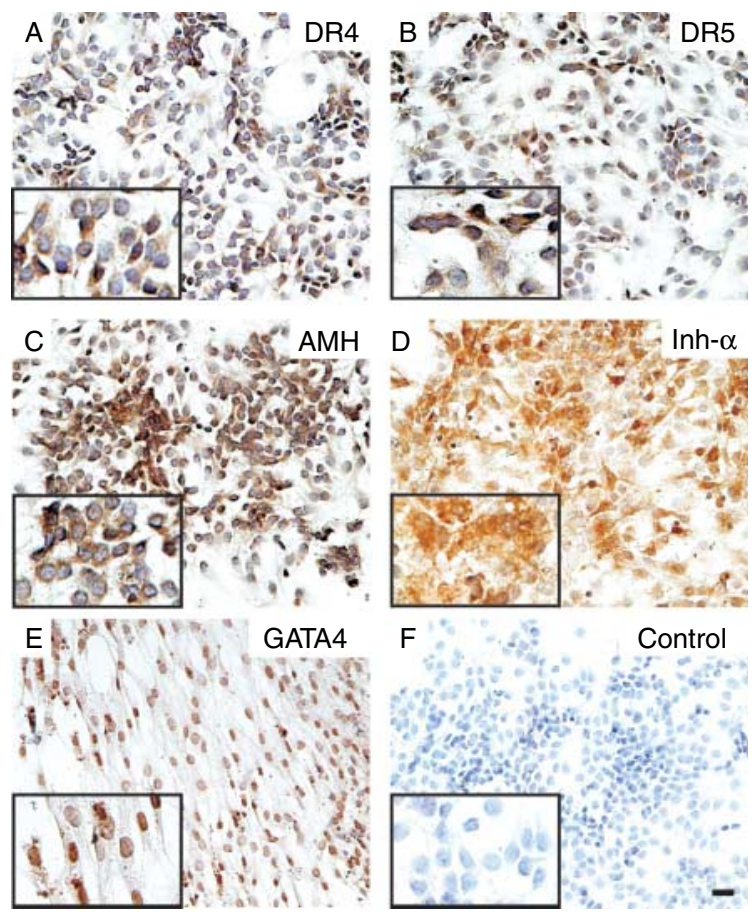

Figure 2 Immunohistochemical analysis of TRAIL receptors DR4 (A), DR5 (B), and GCT markers AMH (C), $\alpha$-inhibin (inh- $\alpha$ ) (D), and GATA4 (E) in cultured GCT cells. Brown color indicates positive staining. (F) A negative control using secondary antibody only. Higher magnifications are shown in insets. Scale bar is $50 \mu \mathrm{m}$.

Importantly, 11 of 12 recurrent GCTs exhibited strong or intermediate DR4 expression, and 12 of 12 tumors exhibited strong or intermediate DR5 expression. The DR4 and DR5 expression patterns did not correlate with clinical stage of primary tumor, size of the tumor, atypia, mitotic index, or with recurrence tendency (data not shown).

\section{TRAIL receptor expression in primary GCT cell cultures}

To further assess the functional role of TRAIL-induced apoptosis in human GCTs, we characterized the expression of DR4 and DR5 in primary GCT cell cultures that we had subsequently treated with TRAIL (see below). For in vitro experiments, we obtained six tumor samples, two from primary tumors and four from recurrent tumors. All the samples of primary GCTs studied expressed the TRAIL receptors DR4 (Fig. 2A) and DR5 (Fig. 2B).

The successful isolation of the tumor cells was confirmed by immunostaining for GCT markers as follows: AMH, inhibin- $\alpha$, and GATA4 (Fig. 2C-E; 
Rey et al. 2000, La Marca \& Volpe 2007, Jamieson \& Fuller 2008). Immunocytochemistry results were verified using reverse transcription-PCR analysis of primary GCT cell lysates (data not shown).

\section{TRAIL induces apoptosis in primary GCT cultures but does not affect GATA4 levels}

Recombinant TRAIL has been shown to induce apoptosis in the human GCT cell line KGN (Woods et al. 2008a, Jaaskelainen et al. 2009). To clarify the significance of the TRAIL pathway in GCT pathobiology and apoptosis, we compared the effects of TRAIL in primary human GCT cells and in the KGN cell line. Freshly isolated primary GCT cells and KGN cells were stimulated with increasing doses of TRAIL for $24 \mathrm{~h}$ before the measurement of caspase activity. Analysis of nuclear morphology (based on DAPI staining) was utilized to confirm the induction of apoptosis. In these experiments, TRAIL dose dependently activated caspase- 3 and induced apoptosis in all the six GCTs studied (Fig. 3A and B). Activation of caspase-3 was also evident in KGN cells (Fig. 3A),

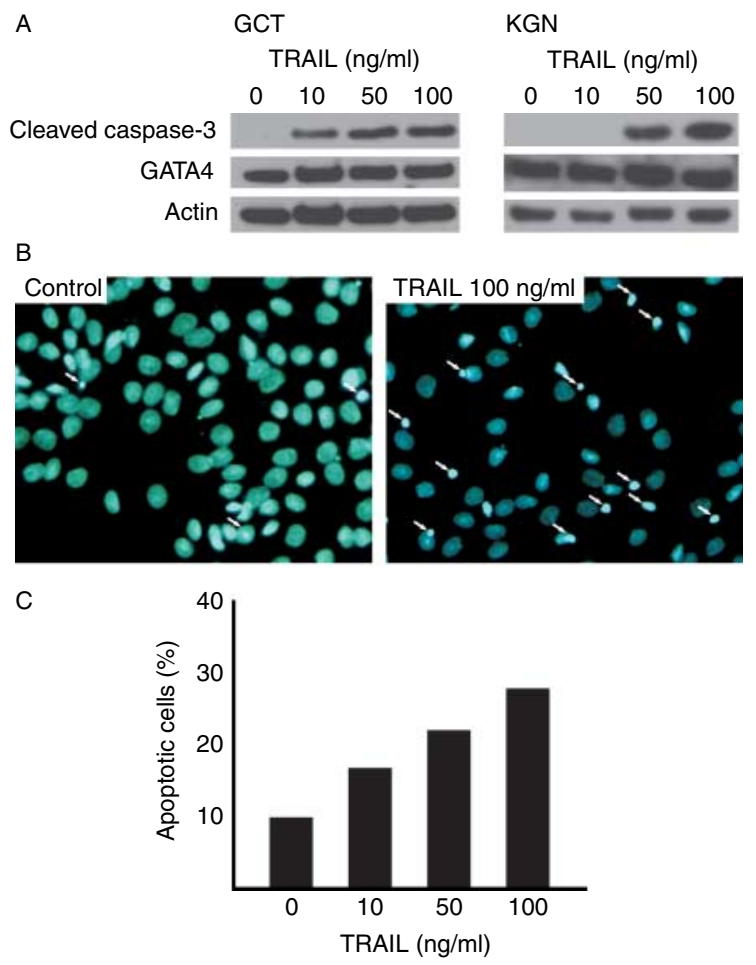

Figure 3 (A) Western blotting showing the activation of caspase- 3 in both primary GCT and KGN cells $24 \mathrm{~h}$ after TRAIL administration. Expression of GATA4 remains unaltered. $\beta$-Actin served as a loading control. (B) DAPI staining of apoptotic primary GCT cells $24 \mathrm{~h}$ after TRAIL administration. Arrows indicate apoptotic cells. (C) A representative histogram of dose-dependent activation of GCT apoptosis by TRAIL. confirming the similar behavior between KGN cell line and primary human GCT cells. However, the primary GCT cells were more sensitive to TRAIL-induced apoptosis as activated caspase-3 was detected in TRAIL concentrations as low as $10 \mathrm{ng} / \mathrm{ml}$. This difference may reflect the more robust nature of established cell line, $\mathrm{KGN}$, or the greater cellular stress involved in obtaining the GCT cells from tumor samples.

We next studied the effect of TRAIL administration on GATA4 expression in GCT-derived cells. In human granulosa cells, rapid downregulation of GATA4 precedes the physiological apoptosis of ovulating follicles suggesting its role in the regulation of granulosa cell apoptosis (Heikinheimo et al. 1997). Furthermore, high GATA4 expression is known to correlate with the aggressive behavior of GCT tumors (Anttonen et al. 2005). However, in our experiments, GATA4 protein levels after TRAIL administration changed in neither the primary tumor cells nor the KGN cells (Fig. 3A).

Taken together, these results indicate the presence of functional extrinsic apoptosis pathway in primary GCT cells, and the ability of TRAIL to induce apoptosis in GCTs similar to other ovarian cancer types (Cuello et al. 2001).

\section{Overexpression of GATA4 protects GCT cells from TRAIL-induced apoptosis}

Next, we studied the effect of GATA4 on TRAILinduced GCT apoptosis by assessing the effect of GATA4 overexpression on TRAIL-induced apoptosis in KGN cells using an adenoviral GATA4 construct. KGN cells were chosen instead of primary tumor cells to allow enough experimental material for replicated assays. Consistent with prior studies (Jaaskelainen et al. 2009), TRAIL induced apoptosis in KGN cells in a similar way to primary GCT cell cultures (Figs 3 and $4, P<0.05$ ).

GATA4 overexpression efficiently protected KGN cells from TRAIL-induced apoptosis (Fig. 4). Of note, GATA4 was able to counteract even high doses of TRAIL $(100 \mathrm{ng} / \mathrm{ml})$ after both $6 \mathrm{~h}$ (Fig. 4B; $P<0.05)$ and $24 \mathrm{~h}$ (Fig. 4C; $P<0.05$ ) of stimulation. Overexpression of GATA4 did not affect the baseline caspase activity of KGN cells (Fig. 4B and C). The results are shown in relative caspase activity levels to maintain uniformity between individual adenoviral transfection since individual transfections showed some fluctuation (7000 fluorescence units \pm 2031 ). Importantly, however, normalization to baseline apoptosis rates yielded similar results. 
A

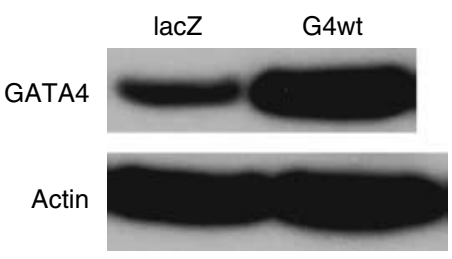

B
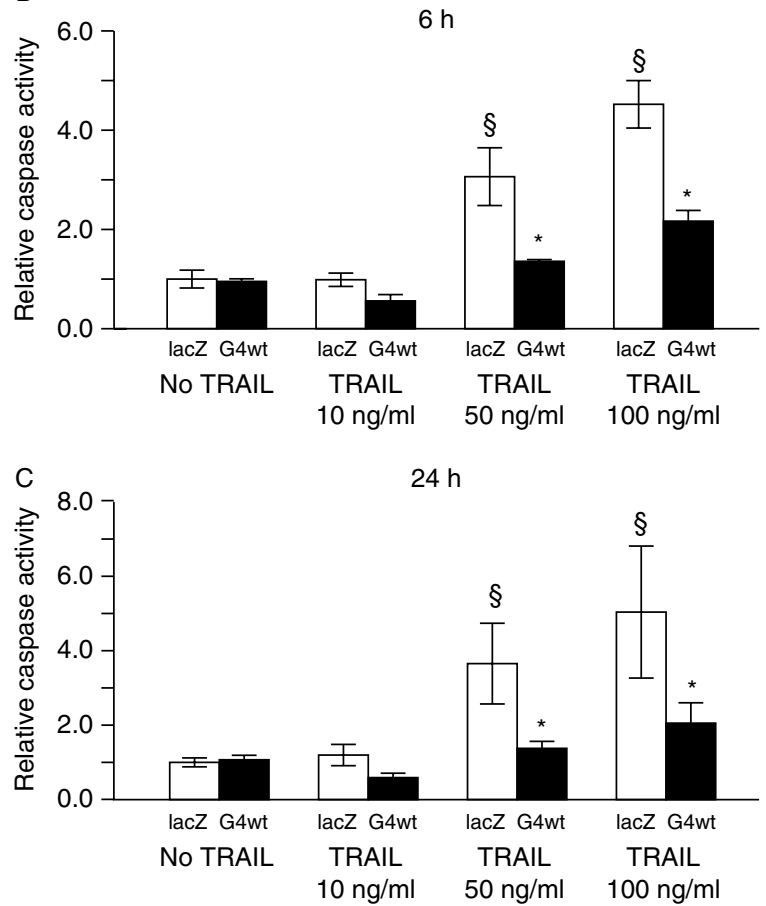

Figure 4 GATA4 protects KGN cells against TRAIL-induced apoptosis. (A) Western blotting showing overexpression of GATA4. The KGN cells were transfected with GATA4 adenovirus (G4wt) or lacZ control adenovirus (lacZ), and treated with vehicle or indicated doses of TRAIL $48 \mathrm{~h}$ later. Caspase activity was measured $6 \mathrm{~h}(\mathrm{~B})$ and $24 \mathrm{~h} \mathrm{(C)}$ after TRAIL administration. Caspase activity is presented relative to control transfection as the mean \pm S.E.M. of three independent experiments performed in triplicate. ${ }^{*} P<0.05$ as compared to lacZ controls with the same TRAIL concentration. ${ }^{\S} P<0.05$ as compared to lacZ control with no TRAIL.

\section{Disrupting GATA4 function induces apoptosis in GCT cells and potentiates the effect of TRAIL administration}

To further evaluate the role of GATA4 in TRAILinduced GCT apoptosis, we investigated the effect of GATA4 downregulation on GCT cell apoptosis. For this purpose, we transduced KGN cells with lentiviral vectors expressing two different shGATA4 (Fig. 5A). The silencing of GATA4 by either of these shRNAs enhanced the effect of TRAIL-induced apoptosis (Fig. 5B). Despite a relatively modest downregulation of GATA4, these experiments showed increased baseline apoptosis of KGN cells.
To gain better insight into this process, we transfected KGN cells with GATA4-engrailed fusion protein $(\mathrm{G} 4 \mathrm{dn})$, which is known to have a G4dn activity (Liang et al. 2001). Disrupting GATA4 function with the dominant negative mutant markedly increased baseline apoptosis in KGN cells and sensitized these cells to TRAIL-induced apoptosis (Fig. 5D).

A
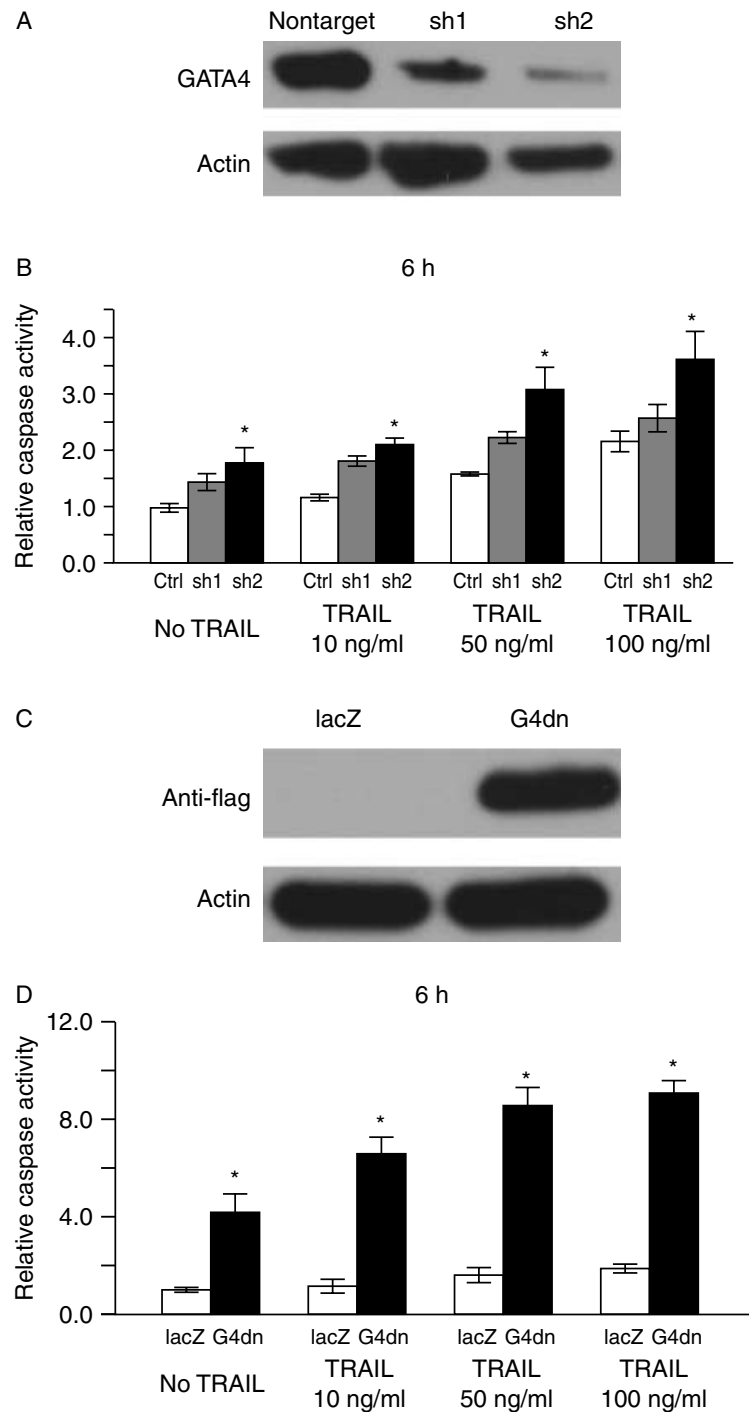

Figure 5 Effect of shGATA4 and dominant negative GATA4 on KGN apoptosis. (A) Western blotting showing silencing of GATA4 in KGN cells expressing two different shGATA4 constructs (sh1 and sh2). (B) The KGN cells stably expressing shGATA4 were treated with vehicle or indicated doses of TRAIL. Caspase activity was measured $6 \mathrm{~h}$ later. (C) Western blotting showing expression of dominant negative GATA4 (G4dn) detected by anti-flag antibody. (D) The KGN cells were transfected with G4dn or control adenovirus, and treated with vehicle or indicated doses of TRAIL. Caspase activity and protein levels were measured $6 \mathrm{~h}$ later. All caspase activities are presented relative to control transfection as the mean \pm S.E.M. of three independent experiments performed in triplicate. ${ }^{*} P<0.05$. 
The increase in apoptosis with G4dn exceeded that obtained by shGATA4, probably because of a relatively modest downregulation effect of shGATA4, 42\% (for Sh1) and 35\% (for Sh2) as compared to non-target controls. Another potential explanation for a larger effect seen with G4dn transfections could be the effect of this construct on nuclear factor $\kappa \mathrm{B}(\mathrm{NF}-\kappa \mathrm{B})$ levels. We did not, however, detect any effects of G4dn transfection on NF- $\kappa \mathrm{B}$ protein levels when evaluated by western blot analysis (data not shown). Taken together, these results suggest that GATA4 functions as an antiapoptotic factor in GCTs.

\section{Discussion}

Recombinant human TRAIL and antibodies mimicking its apoptosis-inducing effect have emerged as promising drugs to treat variety of malignant tumors. Currently, phase II clinical studies are being conducted to study this approach in the treatment of both advanced colorectal and non-small cell lung cancer as well as relapsed non-Hodgkin's lymphoma (Ashkenazi 2008).

A prerequisite for TRAIL-based therapy is the expression of death receptors on the cancer cell surface (Ashkenazi 2008). We now show that both TRAIL death receptors DR4 and DR5 are present in a majority of human GCTs. We also report that TRAIL receptors, and the other components of TRAIL pathway, are functional in primary isolated GCT cells. Taken together, these preclinical studies provide a basis to test the use of TRAIL as an anti-cancer drug also in human GCTs. Since TRAIL receptors are present in normal human granulosa cells, further studies are needed to assess the sensitivity of these cells to TRAIL-induced apoptosis.

Besides the extrinsic pathway via death receptors, apoptosis is also regulated by the intrinsic pathway. Indeed, promising combination therapies targeting both these pathways are being studied in different malignancies (Johnstone et al. 2008). In physiological granulosa cells, genes of $\mathrm{Bcl}-2$ family are known to play key role in the regulation of the intrinsic apoptosis pathway (Pru \& Tilly 2001). In animal studies, $B c l-2$ knockout mice demonstrate decreased number of follicles (Ratts et al. 1995), whereas granulosa cell apoptosis is compromised in transgenic mice overexpressing Bcl-2 (Hsu et al. 1996). Intriguingly, the transcription factor GATA4 is known to regulate the expression of $\mathrm{Bcl}-2$ in human GCTs (Kyronlahti et al. 2008).

Transcription factor GATA4 is best known for its role in heart muscle development and function (Heikinheimo et al. 1994). Mice devoid of
GATA4 die in utero due to disturbances in cardiac morphogenesis (Kuo et al. 1997, Molkentin et al. 1997). Recently, GATA4 has emerged as an important factor in the development and function of endocrine tissues (Viger et al. 2008). In adult cardiomyocytes, GATA4 acts as a pivotal anti-apoptotic factor during different apoptosis inducing stimuli such as hypoxemia (Heineke et al. 2007) or treatment with anti-cancer drug doxorubicin (Kobayashi et al. 2006, 2009).

In this study, we have tested the ability of GATA4 to modify TRAIL-induced apoptosis in human GCTs. Treating KGN cells with TRAIL did not have any effect on endogenous GATA4 levels in GCT cells. Thus, the apoptosis-inducing effect of TRAIL does not require the downregulation of GATA4. Overexpression of GATA4 partially protects KGN cells from TRAIL-induced apoptosis. The anti-apoptotic effect of GATA4 in GCT cells is of interest given that the tumors with high GATA4 expression are aggressive and relapse more often and earlier than tumors with low GATA4 expression (Anttonen et al. 2005). In tumors of high expression, elevated levels of GATA4 may therefore protect malignant cells from the apoptotic effects of endogenous factors such as TRAIL. The role of GATA4 in GCT pathobiology is strengthened by our findings that disturbing GATA4 by either dominant negative adenovirus or shRNAproducing constructs sensitizes KGN cells to TRAILinduced apoptosis. As a next step, injection of cell lines with increased or silenced GATA4 expression to SCID mouse models could be utilized to assess the interplay between recombinant TRAIL and sensitizing treatments. Combining the stimulation of the extrinsic TRAIL-dependent pathway to sensitizing treatments may be useful in treating GCTs.

Interestingly, disrupting GATA4 function with either dominant negative adenovirus or shGATA4 lentivirus induced apoptosis in GCTs even in the absence of exogenous TRAIL supporting the premise that GATA4 functions as an anti-apoptotic factor in GCTs. The induction of apoptosis after GATA4 downregulation was of the same extent with all the TRAIL concentrations tested and even without TRAIL stimulation, indicating that the effect of GATA4 knockdown is not specific for TRAIL stimulations. GATA4 may thus modify multiple apoptosis-inducing pathways in granulosa cells. This observation is in accordance with the findings in normal ovary, in which downregulation of GATA4 precedes the physiological apoptosis of ovulating follicles (Heikinheimo et al. 1997). It is not known how GATA4 exerts its antiapoptotic effects in the ovary, but it is known to regulate the two anti-apoptotic Bcl-2 family members, 
namely $\mathrm{Bcl}-2$ and $\mathrm{Bcl}-\mathrm{Xl}$, in GCT cells as well as in cardiomyocytes by directly binding to their promoters (Kitta et al. 2003, Kobayashi et al. 2006, Kyronlahti et al. 2008). Thus, proteins in the Bcl-2 family appear as putative candidates to mediate the GATA4 effects in human GCTs.

In summary, our studies document the expression of functional TRAIL receptors in GCTs. Furthermore, we show that GATA4 protects GCT cells from TRAILinduced apoptosis in vitro, whereas inhibiting the normal GATA4 function predisposes GCT cells to TRAIL-induced apoptosis. Finally, we show that disturbing GATA4 function alone is sufficient to induce GCT apoptosis in vitro, suggesting that GATA4 may function as a survival factor in GCTs.

\section{Declaration of interest}

The authors declare that there is no conflict of interest that could be perceived as prejudicing the impartiality of the research reported.

\section{Funding}

This work was supported by Academy of Finland (to M Anttonen and M Heikinheimo), Finnish Cancer Organizations (to M Heikinheimo), Helsinki Biomedical Graduate School (to A Kyrönlahti), and Helsinki University Research Funds (to L Unkila-Kallio, R Butzow, and M Heikinheimo).

\section{Acknowledgements}

We thank Drs Yoshihiro Nishi and Toshihiko Yanase for the KGN cell line, and Drs Jeffery Molkentin, Mary HunzikerDunn, and Qiangrong Liang for adenoviral constructs. We also thank Ms Taru Jokinen and Ms Teija Karkkulainen for excellent technical assistance, as well as gynecologists and operation room staff at Department of Obstetrics and Gynecology, Helsinki University Central Hospital for help with primary tumor samples.

\section{References}

Anttonen M, Unkila-Kallio L, Leminen A, Butzow R \& Heikinheimo M 2005 High GATA-4 expression associates with aggressive behavior, whereas low anti-Mullerian hormone expression associates with growth potential of ovarian granulosa cell tumors. Journal of Clinical Endocrinology and Metabolism 90 6529-6535.

Ashkenazi A 2008 Directing cancer cells to self-destruct with pro-apoptotic receptor agonists. Nature Reviews. Drug Discovery 7 1001-1012.

Charron F, Tsimiklis G, Arcand M, Robitaille L, Liang Q, Molkentin JD, Meloche S \& Nemer M 2001 Tissuespecific GATA factors are transcriptional effectors of the small GTPase RhoA. Genes and Development 15 2702-2719.
Colombo N, Parma G, Zanagnolo V \& Insinga A 2007 Management of ovarian stromal cell tumors. Journal of Clinical Oncology 25 2944-2951.

Cuello M, Ettenberg SA, Nau MM \& Lipkowitz S 2001 Synergistic induction of apoptosis by the combination of TRAIL and chemotherapy in chemoresistant ovarian cancer cells. Gynecologic Oncology 81 380-390.

Fulda S \& Debatin KM 2006 Extrinsic versus intrinsic apoptosis pathways in anticancer chemotherapy. Oncogene 25 4798-4811.

Fuller PJ \& Chu S 2004 Signalling pathways in the molecular pathogenesis of ovarian granulosa cell tumours. Trends in Endocrinology and Metabolism 15 122-128.

Heikinheimo M, Scandrett JM \& Wilson DB 1994 Localization of transcription factor GATA-4 to regions of the mouse embryo involved in cardiac development. Developmental Biology 164 361-373.

Heikinheimo M, Ermolaeva M, Bielinska M, Rahman NA, Narita N, Huhtaniemi IT, Tapanainen JS \& Wilson DB 1997 Expression and hormonal regulation of transcription factors GATA-4 and GATA-6 in the mouse ovary. Endocrinology 138 3505-3514.

Heineke J, Auger-Messier M, Xu J, Oka T, Sargent MA, York A, Klevitsky R, Vaikunth S, Duncan SA, Aronow BJ et al. 2007 Cardiomyocyte GATA4 functions as a stress-responsive regulator of angiogenesis in the murine heart. Journal of Clinical Investigation 117 3198-3210.

Hsu SY, Lai RJ, Finegold M \& Hsueh AJ 1996 Targeted overexpression of Bcl-2 in ovaries of transgenic mice leads to decreased follicle apoptosis, enhanced folliculogenesis, and increased germ cell tumorigenesis.

Endocrinology 137 4837-4843.

Jaaskelainen M, Kyronlahti A, Anttonen M, Nishi Y, Yanase T, Secchiero P, Zauli G, Tapanainen JS, Heikinheimo M \& Vaskivuo TE 2009 TRAIL pathway components and their putative role in granulosa cell apoptosis in the human ovary. Differentiation 77 369-376.

Jamieson S \& Fuller PJ 2008 Management of granulosa cell tumour of the ovary. Current Opinion in Oncology 20 560-564.

Johnstone RW, Frew AJ \& Smyth MJ 2008 The TRAIL apoptotic pathway in cancer onset, progression and therapy. Nature Reviews. Cancer 8 782-798.

Kitta K, Day RM, Kim Y, Torregroza I, Evans T \& Suzuki YJ 2003 Hepatocyte growth factor induces GATA-4 phosphorylation and cell survival in cardiac muscle cells. Journal of Biological Chemistry 278 4705-4712.

Kobayashi S, Lackey T, Huang Y, Bisping E, Pu WT, Boxer LM \& Liang Q 2006 Transcription factor gata4 regulates cardiac BCL2 gene expression in vitro and in vivo. FASEB Journal 20 800-802.

Kobayashi S, Volden P, Timm D, Mao K, Xu X \& Liang Q 2009 Transcription factor GATA4 inhibits doxorubicininduced autophagy and cardiomyocyte death. Journal of Biological Chemistry 285 793-804.

Kononen J, Bubendorf L, Kallioniemi A, Barlund M, Schraml P, Leighton S, Torhorst J, Mihatsch MJ, Sauter G 
\& Kallioniemi OP 1998 Tissue microarrays for highthroughput molecular profiling of tumor specimens. Nature Medicine 4 844-847.

Kuo CT, Morrisey EE, Anandappa R, Sigrist K, Lu MM, Parmacek MS, Soudais C \& Leiden JM 1997 GATA4 transcription factor is required for ventral morphogenesis and heart tube formation. Genes and Development 11 1048-1060.

Kyronlahti A, Ramo M, Tamminen M, Unkila-Kallio L, Butzow R, Leminen A, Nemer M, Rahman N, Huhtaniemi I, Heikinheimo M et al. 2008 GATA-4 regulates Bcl-2 expression in ovarian granulosa cell tumors. Endocrinology 149 5635-5642.

La Marca A \& Volpe A 2007 The anti-Mullerian hormone and ovarian cancer. Human Reproduction Update 13 265-273.

Liang Q, De Windt LJ, Witt SA, Kimball TR, Markham BE \& Molkentin JD 2001 The transcription factors GATA4 and GATA6 regulate cardiomyocyte hypertrophy in vitro and in vivo. Journal of Biological Chemistry 276 30245-30253.

Molkentin JD, Lin Q, Duncan SA \& Olson EN 1997 Requirement of the transcription factor GATA4 for heart tube formation and ventral morphogenesis. Genes and Development 11 1061-1072.

Nishi Y, Yanase T, Mu Y, Oba K, Ichino I, Saito M, Nomura M, Mukasa C, Okabe T, Goto K et al. 2001 Establishment and characterization of a steroidogenic human granulosalike tumor cell line, KGN, that expresses functional follicle-stimulating hormone receptor. Endocrinology 142 437-445.

Partanen JI, Nieminen AI, Makela TP \& Klefstrom J 2007 Suppression of oncogenic properties of c-Myc by LKB1controlled epithelial organization. PNAS $\mathbf{1 0 4}$ 14694-14699.

Pru JK \& Tilly JL 2001 Programmed cell death in the ovary: insights and future prospects using genetic technologies. Molecular Endocrinology 15 845-853.
Ratts VS, Flaws JA, Kolp R, Sorenson CM \& Tilly JL 1995 Ablation of bcl-2 gene expression decreases the numbers of oocytes and primordial follicles established in the postnatal female mouse gonad. Endocrinology 136 3665-3668.

Rey R, Sabourin JC, Venara M, Long WQ, Jaubert F, Zeller WP, Duvillard P, Chemes H \& Bidart JM 2000 Anti-Mullerian hormone is a specific marker of sertoliand granulosa-cell origin in gonadal tumors. Human Pathology 31 1202-1208.

Schrader KA, Gorbatcheva B, Senz J, Heravi-Moussavi A, Melnyk N, Salamanca C, Maines-Bandiera S, Cooke SL, Leung P, Brenton JD et al. 2009 The specificity of the FOXL2 c.402C $>\mathrm{G}$ somatic mutation: a survey of solid tumors. PLoS ONE 4 e7988.

Schumer ST \& Cannistra SA 2003 Granulosa cell tumor of the ovary. Journal of Clinical Oncology 21 1180-1189.

Shah SP, Kobel M, Senz J, Morin RD, Clarke BA, Wiegand KC, Leung G, Zayed A, Mehl E, Kalloger SE et al. 2009 Mutation of FOXL2 in granulosa-cell tumors of the ovary. New England Journal of Medicine 360 2719-2729.

Tilly JL 1996 Apoptosis and ovarian function. Reviews of Reproduction 1 162-172.

Viger RS, Guittot SM, Anttonen M, Wilson DB \& Heikinheimo M 2008 Role of the GATA family of transcription factors in endocrine development, function, and disease. Molecular Endocrinology 22 781-798.

Woods DC, Alvarez C \& Johnson AL 2008a Cisplatinmediated sensitivity to TRAIL-induced cell death in human granulosa tumor cells. Gynecologic Oncology 108 632-640.

Woods DC, Liu HK, Nishi Y, Yanase T \& Johnson AL $2008 b$ Inhibition of proteasome activity sensitizes human granulosa tumor cells to TRAIL-induced cell death. Cancer Letters 260 20-27. 